\title{
Editorial
}

\section{Are We Able to Differentiate between True Mental Disorders and Homeostatic Reactions to Adverse Life Events?}

\author{
Mario Maj \\ Department of Psychiatry, University of Naples SUN, Naples, Italy
}

'Determining when relatively common experiences such as anxiety or sadness... should be considered evidence of some disorder requires the setting of boundaries that are largely arbitrary, not scientific, unlike setting the boundaries for what constitutes cancer or pneumonia.' This is a statement which appeared a few years ago in a vitriolic book entitled Making Us Crazy. DSM: The Psychiatric Bible and the Creation of Mental Disorders [1]. To the average psychiatrist, this statement will probably appear incorrect and ideologically biased. He has learnt to believe that our current systems for the diagnosis and classification of mental disorders have received a convincing empirical validation and that these systems differentiate reliably between true mental disorders and common hassles of everyday life.

'It is not always easy to establish a threshold for a mental disorder, particularly in light of how common symptoms of mental distress are and the lack of objective, physical symptoms.' This is a statement by the US Surgeon General [2]. Here, ignorance and ideological bias are not likely to be involved. However, this view from outside the psychiatric field could still reflect to some degree a prejudice against our profession.

'Based on the high prevalence rates identified in both the ECA and the NCS, it is reasonable to hypothesize that some syndromes in the community represent transient homeostatic responses to internal or external stimuli that do not represent true psychopathologic disorders.' This is a statement coming from within the psychiatric field, and from a very authoritative source [3]. Perhaps at this point the average psychiatrist will have realized that the problem is a real one: at the moment we do not have a clear idea of how to fix the threshold for the diagnosis of some mental disorders (in particular of those with the highest prevalence in the community, i.e. mood and anxiety disorders), and of how to differentiate between these disorders and homeostatic reactions to adverse life events. We have to address this situation urgently, because it may damage the credibility of our discipline and our profession.

Why is this problem becoming so visible only now? One contributing factor has certainly been the recent broadening of the scope of psychiatric intervention from traditional hospital settings, where the issue whether admitted patients had or did not have a mental disorder was not really relevant, to community settings, where this issue is a sensitive one in several cases. A second factor has been the increased presence and influence in the mental health field of several other professions, whose perception of mental health problems is often different from that of psychiatrists. A third factor has been the higher level of information and awareness of users, families and the public opinion. A fourth factor has probably been the translation of traditional descriptive definitions of mental disorders into current operational diagnostic criteria. Traditional definitions seemed to convey a gestalt, a structure of the various disorders, while current operational definitions seem to be less able to do so. Whether

\section{KARGER}

Fax +4161306 1234

E-Mail karger@karger.ch

www.karger.com (c) $2007 \mathrm{~S}$. Karger AG, Basel

0033-3190/07/0765-0257\$23.50/0

Accessible online at:

www.karger.com/pps
Mario Maj

Department of Psychiatry, University of Naples SUN

Largo Madonna delle Grazie

IT-80138 Naples (Italy)

Tel. +39081566 6502, Fax +390815666523, E-Mail majmario@tin.it 
that gestalt was a reality or an illusion remains open to debate [4], but the threshold for the diagnosis of some mental disorders appears more clearly arbitrary today than in the past.

How can we approach this problem? Let me summarize three possible approaches.

The first approach is the one emphasizing the context in which the symptoms occur. The DSM-III and its successors have deliberately tried to exclude the assessment of the context from the diagnostic process. However, the context in which the symptoms occur may be crucial for the distinction between a true mental disorder and a homeostatic reaction, in particular between a true depression and an understandable reaction to a severe adverse event. This is what Wakefield [5] has pointed out, proposing that the diagnosis of depression be 'excluded if the sadness response is caused by a real loss that is proportional in magnitude to the intensity and duration of the response'. This approach looks reasonable, but has its obvious limitations. The decision on whether there is a causal relationship between the event and the response and on whether the response is proportional to the event would be left to the subjective, value-laden [6] judgement of the clinician, with a high risk of low reliability. What is a 'normal' response? Is there a 'normal' response to a given stressor which is the same for all individuals in all cultures?

A second approach to the problem is the one emphasizing possible 'qualitative' differences between true mental disorders and homeostatic reactions to adverse events. This brings us back to the above-mentioned issue of the translation of traditional definitions of mental disorders into operational terms. Did we lose something in this translation? Did any oversimplification of psychopathology or reduction of complex phenomena to their minimum common denominator occur in the search for more precise, concise and reliable diagnostic criteria? I raised this issue a few years ago concerning the concept of schizophrenia [4], while Helmchen and Linden [7] have raised it for the concept of depression: 'Normal forms of negative mood such as... despair or sadness must not be mistaken as depressed mood, characterized by a lack of holothymia and being an emotional feeling only known to depressed persons'. Indeed, some recent studies have revived the research line exploring the nature of the 'distinct quality of mood' which differentiates at least some forms of depression from understandable sadness. In one of these studies [8], patients with a clinical diagnosis of major depression were asked to describe in their own words their depressed mood. The commonest descrip- tion was of 'the experience of lethargy and inability to do things, whether because of tiredness, a specific inability to summon up effort, a feeling of being inhibited or an inability to envisage the future'. The second most common description was of 'a sense of detachment from the environment'. The third description was of 'a feeling that the subject was coming down with a viral illness, either influenza or glandular fever, along with aches and pains and, in particular, headaches or numbness of the head or tight bands around the head.' Sadness and unhappiness did not appear at all in the descriptions. To what extent can these findings be generalized? Is this 'distinct' quality of mood a fact, at least in some forms of depression, or an illusion which we will never be able to translate into operational terms?

The third approach to the problem is the one assuming that the boundary between some mental disorders and homeostatic reactions to adverse events is unavoidably arbitrary and has to be decided on pragmatic grounds [9]. This is the principle underlying the recent attempt to correct the results of the Epidemiological Catchment Area Study and the National Comorbidity Survey by introducing the 'clinical significance' criterion ('the respondent's symptoms led him or her to mention them to a doctor or other professional, or the symptoms interfered with his or her everyday life, or the respondent took medication for the symptoms') [10]. This criterion, however, does not really discriminate between true mental disorders and transient homeostatic reactions, because the latter can interfere significantly with everyday life, be brought to the attention of mental health professionals, and lead to the prescription (or self-prescription) of psychotropic drugs. As pointed out by Engel [11] almost 50 years ago, the "normal' or 'natural' response to the loss of a valued object (not only of a loved person, but also of a job, status, home, an ideal, a part of the body, etc.) may involve an impairment of the capacity to function which may last for weeks and even months.

So, it is clear that the issue of the differential diagnosis between some mental disorders (in particular mood and anxiety disorders) and homeostatic reactions to life events is at present completely open. Further research aimed to provide empirical support to one of the above-mentioned (or other alternative) approaches is obviously warranted. In the absence of new research data, it is likely that 'threshold' issues will be increasingly ignored in the future, and that sequential stepwise treatment algorithms for people with specific symptoms (irrespective of their diagnosis) will become increasingly popular. 


\section{References}

1 Kutchins H, Kirk SA: Making Us Crazy. DSM: The Psychiatric Bible and the Creation of Mental Disorders. New York, Free Press, 1997.

2 U.S. Department of Health and Human Services: Mental Health: A Report of the Surgeon General. Executive Summary. Rockville, National Institutes of Health, National Institute of Mental Health, 1999.

3 Regier DA, Kaelber CT, Rae DS, Farmer ME, Knauper B, Kessler RC, Norquist GS: Limitations of diagnostic criteria and assessment instruments for mental disorders: implications for research and policy. Arch Gen Psychiatry 1998;55:109-115.
4 Maj M: Critique of the DSM-IV operational diagnostic criteria for schizophrenia. Br J Psychiatry 1998;172:458-460.

5 Wakefield JC: Diagnosing DSM-IV. 1. DSMIV and the concept of disorder. Behav Res Ther 1997;35:633-649.

6 Fulford KWM, Broome M, Stanghellini G, Thornton T: Looking with both eyes open: fact and value in psychiatric diagnosis? World Psychiatry 2005;4:78-86.

7 Helmchen H, Linden M: Subthreshold disorders in psychiatry: clinical reality, methodological artefact, and the double-threshold problem. Compr Psychiatry 2000;41:1-7.

8 Healy D: Dysphoria; in Costello CG (ed): Symptoms of Depression. Chichester, Wiley, 1993, pp 24-45.
9 Kendell R, Jablensky A: Distinguishing between the validity and utility of psychiatric diagnoses. Am J Psychiatry 2003;160:4-12.

10 Narrow WE, Rae DS, Robins LN, Regier DA: Revised prevalence estimates of mental disorders in the United States: using a clinical significance criterion to reconcile 2 surveys estimates. Arch Gen Psychiatry 2002;59: 115-123.

11 Engel GL: Is grief a disease? A challenge for medical research. Psychosom Med 1961;23: $18-22$. 